\title{
Combined In Situ and Ex Situ Study on Synthesis of Nanostructured Catalyst in Solid State
}

\author{
Wenpei $\mathrm{Gao}^{1}$, Christopher Addiego ${ }^{2}$, Yanling $\mathrm{Ma}^{3}$, Jianbo $\mathrm{Wu}^{3}$ and Xiaoqing $\mathrm{Pan}^{1,2}$ \\ ${ }^{1 .}$ Department of Chemical Engineering and Materials Science, University of California, Irvine, Irvine, \\ CA. \\ 2. Department of Physics and Astronomy, University of California, Irvine, Irvine, CA. \\ 3. School of Materials Science and Engineering, Shanghai Jiao Tong University, Shanghai, China.
}

One dimensional (1D) nanomaterials start to play an important role in the fabrication of electronic, optoelectronic and electrochemical devices due to their advantageous properties, including enhanced stability, carrier mobility and high structural anisotropy. For example, Pt-based nanowires (NWs) offer enhanced electrocatalytic performance owing to their high flexibility, outstanding conductivity and thermal stability compared with their zero dimensional (0D) NPs counterparts. Available approaches to synthesize Pt-based NWs usually involves reaction in wet chemistry, which hinder the practical scalable application in industry. To this end, growing nanowires in solid state offers a feasible way to make scalable synthesis of such 1D nanostructures.[1]

Using a closed gas cell in a transmission electron microscope (TEM), we were able to study the growth mechanism of nanostructures in solid state at the atomic scale in various gas environments. The in situ TEM imaging was carried out on a Jeol JEM-300CF S/TEM and an FEI Titan microscope, both operated at $300 \mathrm{kV}$. The Protochips Atmosphere gas cell holders were used for the in situ experiments. $\mathrm{K}_{2} \mathrm{PtCl}_{4}$ was dispersed in methanol and sonicated before re-dispersing onto the heating e-chip of the gas cell. During the observation, the gas cell was flushed by Ar gas three times, and heated from room temperature to the desired temperature up to $300{ }^{\circ} \mathrm{C}$ using $1{ }^{\circ} \mathrm{C} / \mathrm{s}$ increments. Afterwards, targeted gas was introduced into the gas cell at the pressure of $1 \mathrm{~atm}$.

Our results show the sintering process is dominant in gas phase reaction. Small particles approach each other via self-diffusion, adhere and finally grow into a solid crystallite. Under most gas environments, the particles attach to each other without any preferential orientation relationship. However, in $\mathrm{H}_{2}$, the nanoparticles approach and attach to each other only along specific orientations, and gradually form short nanowires. As shown in Figure 1a, at $20 \mathrm{~s}$, the three NPs are not in the same orientation according to the Pt $\{111\}$ fringes, indicated by the red lines. At $80 \mathrm{~s}$, the upper Pt NP rotates to re-orient its $\{100\}$ surface planes to match the middle NP; this is followed by attachment of the particles at $240 \mathrm{~s}$ and coalescence at 320 s. the same process also occurs on the bottom nanoparticle. In ex situ experiment, we also demonstrated the successful synthesis of extended long Pt and PtNi nanowires in solid state assisted by $\mathrm{H}_{2}$, as shown in the TEM image in Figure 1b. The atomic resolution high-angle annular dark field (HAADF) STEM image in Figure 1c reveals a nanowire with the side surface corresponding to Pt $\{111\}$ planes in the projection along the zone axis of $\mathrm{Pt}$ (110). Compared to the reported methods on growth of Pt-based NWs, this synthesis approach is free of organic solvent or capping agent, thus eliminating the surface contamination. The resulting catalysts demonstrated enhanced electrocatalytic activity towards ORR performance: the as-prepared Pt-based nanowires deliver the mass activity of $0.71 \mathrm{~A} / \mathrm{mg}_{\mathrm{Pt}}$ for Pt nanowires and $1.52 \mathrm{~A} / \mathrm{mg}_{\mathrm{Pt}}$ for $\mathrm{Pt}_{1.3} \mathrm{Ni}$ nanowires; the specific activity of $2.20 \mathrm{~mA} / \mathrm{cm}^{2}{ }_{\mathrm{Pt}}$ for Pt nanowires and $3.58 \mathrm{~mA} / \mathrm{cm}^{2} \mathrm{Pt}$ for $\mathrm{Pt}_{1.3} \mathrm{Ni}$ nanowires, showing promising improvement over the commercial $\mathrm{Pt} / \mathrm{C}$ catalyst [2]. 
References:

[1] Y. Ma et al, Advanced Materials 29 (46) (2017), p. 1703460.

[2] The electron microscopy work reported here is supported by the NSF Grant No. CBET-1159240 and School of Engineering at University of California, Irvine. Electron microscopy was carried out in Irvine Materials Research Institute, University of California, Irvine, and Center for Nanophase Materials Sciences in Oak Ridge National Laboratory. The authors would like to thank Dr. Miaofang Chi for her help on electron microscopy experiments.

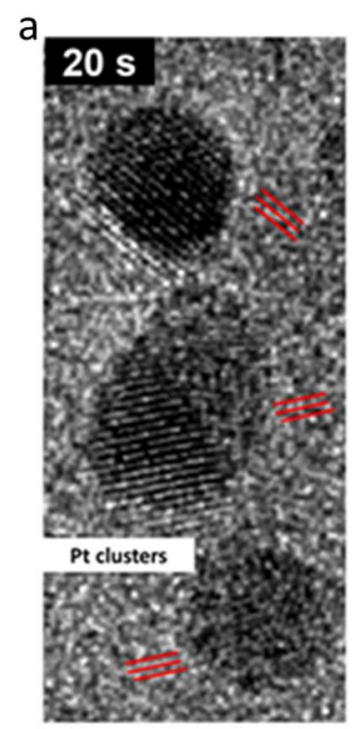

b

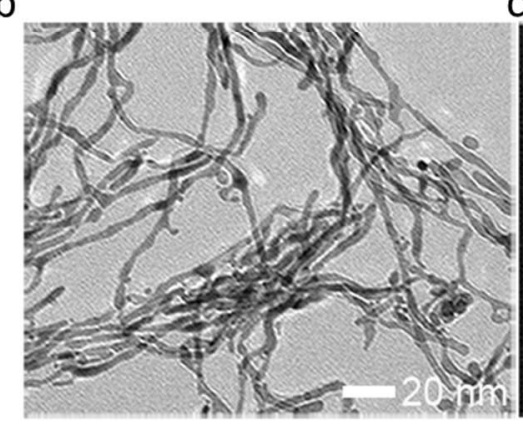

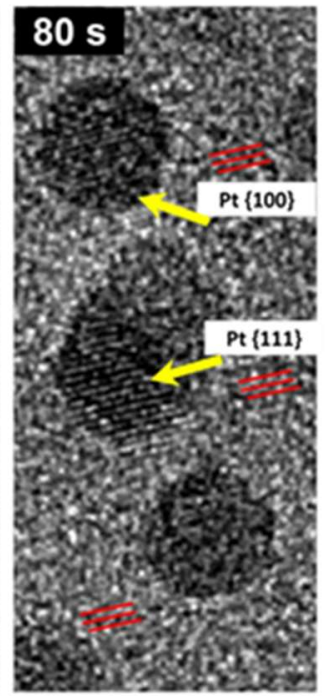

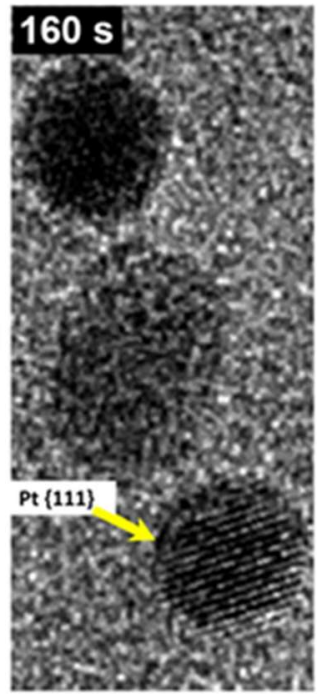

$2 \mathrm{~nm}$

C
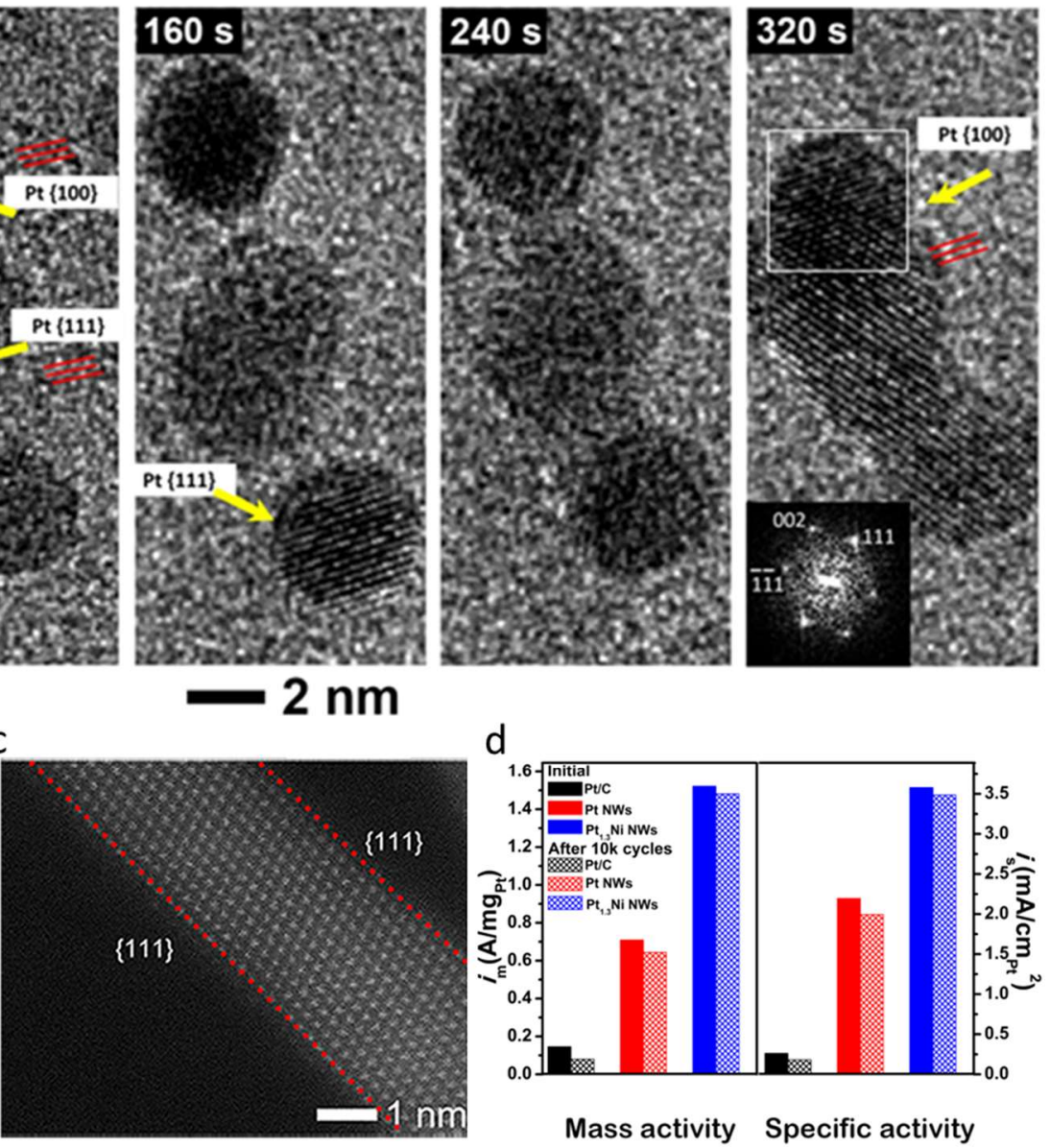

Figure 1

Figure 1. (a) In situ TEM images showing the formation of the nanowire, via oriented-attachment-growth along Pt (100) orientation. The parallel red lines indicate the lattice orientation shared by individual Pt particles before and during attachment. (b) Low-magnification TEM micrograph of a group of $\mathrm{Pt}$ nanowires. (c) Atomic resolution HAADF-STEM image of a nanowire. (d) Electrochemical performance comparison of as-prepared $\mathrm{Pt}$ nanowires and $\mathrm{Pt}_{1.3} \mathrm{Ni}$ nanowires against commercial $\mathrm{Pt} / \mathrm{C}$ Comparison of area-specific activity and mass-specific activity at $0.9 \mathrm{~V}$ vs. RHE before and after 10k cycles. 